\title{
PROVABILITY IN PREDICATE PRODUCT LOGIC
}

\author{
MICHAEL C. LASKOWSKI AND SHIRIN MALEKPOUR
}

\begin{abstract}
We sharpen Hájek's Completeness Theorem for theories extending predicate product logic, $\Pi \forall$. By relating provability in this system to embedding properties of ordered abelian groups we construct a universal BL-chain $\mathbf{L}$ in the sense that a sentence is provable from $\Pi \forall$ if and only if it is an $\mathbf{L}$-tautology. As well we characterize the class of lexicographic sums that have this universality property.
\end{abstract}

\section{INTRODUCTION}

Predicate product logic is a variant of first-order logic wherein sentences are assigned a truth value in the closed interval $[0,1]$. In product logic the truth value of a conjunction $\varphi \& \psi$ of sentences is equal to the product of the truth values of $\varphi$ and $\psi$, which is natural in certain applications (e.g., if $\varphi$ and $\psi$ describe independent events).

In $[\mathrm{H}]$ Petr Hájek laid the groundwork for a proof theory for this logic. He defined an axiom system $\Pi \forall$, which consists of a (recursive) list of 'Basic Logic' axioms BL $\forall$, together with two additional axiom schema:

$$
\begin{gathered}
(\varphi \wedge \neg \varphi) \rightarrow \overline{0} \quad \text { and } \\
\neg \neg \chi \rightarrow((\varphi \& \chi \rightarrow \psi \& \chi) \rightarrow(\varphi \rightarrow \psi))
\end{gathered}
$$

The basic logic axioms are valid in many different predicate fuzzy logic systems, while the two additional axioms are valid in Product Logic (as well as conventional two-valued logic) but distinguish it from Gödel logic and Lukasiewicz logic.

As for semantics, Hájek defined a BL-chain as a linearly ordered residuated lattice satisfying certain properties. Here, however, we follow the treatment set out in [LS1] and [LS2] and define a BL-chain to be an ordered abelian semigroup $(L,+, \leq, 0,1)$ in which 1 is both the maximal element of the ordering and the identity element of the

Partially supported by NSF grant DMS-0300080.

Portions of this material appears in Malekpour's doctoral dissertation. 
semigroup, 0 is the minimal element of the semigroup, and whenever $a<b$ there is a maximal $c$ such that $b+c=a$. By defining $b \Rightarrow a$ to be 1 whenever $b \leq a$ and the maximal $c$ such that $b+c=a$ otherwise, one readily sees that this definition is equivalent to the definition proposed by Hájek in $[\mathrm{H}]$.

Following Hájek, fix a predicate language $\tau$. For a given BL-chain $\mathbf{L}$, an $\mathbf{L}$-structure $\mathbf{M}$ is a triple $\left\langle M,\left(m_{c}\right)_{c},\left(r_{P}\right)_{P}\right\rangle$, where $M$ is a nonempty set, $m_{c}$ is an element of $M$ for each constant symbol $c \in \tau$, and $r_{P}$ : $M^{n} \rightarrow \mathbf{L}$ is a function for every $n$-ary predicate symbol $P \in \tau$. Given an L-structure $\mathbf{M}$, one recursively defines a function $\|\varphi\|_{\mathbf{M}}$ to every $\tau(M)$-sentence $\varphi$ by demanding that $\|\varphi \& \psi\|=\|\varphi\|+\|\psi\| ;\|\varphi \rightarrow \psi\|=$ $\|\varphi\| \Rightarrow\|\psi\| ;\|\exists x \varphi(x)\|=\sup \{\|\varphi(a)\|: a \in M\} ;$ and $\|\forall x \varphi(x)\|=$ $\inf \{\|\varphi(a)\|: a \in M\}$. (In the clauses above, and whenever it is clear, we suppress the subscript on $\|\varphi\|$.) In general, there is no reason why the requisite suprema and infima need exist. Again, following Hájek, we call an $\mathbf{L}$-structure $\mathbf{M}$ safe if in fact all the suprema and infima needed to compute $\|\varphi\|$ do exist for all $\tau(M)$-sentences $\varphi$.

A theory $T$ is simply a set of formulas. We say that an $\mathbf{L}$-structure $\mathbf{M}$ is a model of $T$ if $\|\varphi(\bar{a})\|=1$ for every $\varphi \in T$ and every $\bar{a}$ from $M$ of the requisite length. In $[\mathrm{H}]$ Hájek proves the following results that we use freely:

Theorem 1.1 (Deduction Theorem). For any theory $T$ extending $B L \forall$ and sentences $\varphi, \psi, T \cup\{\varphi\} \vdash \psi$ if and only if $T \vdash \varphi^{k} \rightarrow \psi$ for some positive integer $k$ (where $\varphi^{k}$ denotes the $k$-fold conjunction $\varphi \& \ldots \& \varphi$ ).

Theorem 1.2 (Completeness Theorem). $T \vdash \varphi$ if and only if $\|\varphi\|_{\mathbf{M}}=$ 1 for all BL-chains $\mathbf{L}$ and all safe $\mathbf{L}$-structures $\mathbf{M}$ that model $T$.

In this paper we obtain several strengthenings of Hájek's completeness theorem for theories extending $\Pi \forall$, the strongest of which is Theorem 2.9. As is usual in the study of provability, in order to understand the logical consequences of the theory $\Pi \forall$, one must include nonstandard models as well. In this context, this means defining a class of BL-chains that are generated from ordered abelian groups.

Definition 1.3. Let $(G,+, \leq)$ be any ordered abelian group. Let $N(G)$ be the subsemigroup with universe $\{a \in G: a \leq 0\}$. Let $L(G)$ denote the BL-chain with universe $N(G) \cup\{-\infty\}$ in which + and $\leq$ are inherited from $G$ with the additional stipulations that $-\infty$ is the minimal element of $L(G)$ and that $a+(-\infty)=-\infty$ for all $a \in L(G)$. The 'top' element of $L(G)$ is the zero element of $G$, and the 'bottom' element of $L(G)$ is $-\infty$. 
It is readily verified that $L(G)$ is a BL-chain for any ordered abelian group $G$. (In fact, for any $a<b$ in $L(G)$ there is a unique $c$ such that $b+c=a$.) Furthermore, any $L(G)$-structure is necessarily a model of $\Pi \forall$. In order to describe a converse, we need to specify when two structures can be identified.

Definition 1.4. Fix a predicate language $\tau$. An $\mathbf{L}$-structure $\mathbf{M}$ and an $\mathbf{L}^{\prime}$-structure $\mathbf{M}^{\prime}$ are identified if $M=M^{\prime}, m_{c}^{\mathbf{M}}=m_{c}^{\mathbf{M}^{\prime}}$ for all constant symbols $c \in \tau$, and

$$
\|\varphi\|_{\mathbf{M}}=\|\varphi\|_{\mathbf{M}^{\prime}}
$$

for all $\tau(M)$-formulas $\varphi$.

The intuition behind identifying such structures is that 'extra elements' of $\mathbf{L}$ or $\mathbf{L}$ ' are irrelevant if they never appear in the computation of the truth of any $\tau(M)$-sentence. The following proposition is fundamental to our analysis.

Proposition 1.5. Any L-structure $\mathbf{M}$ that is a model of $\Pi \forall$ (i.e., a model of the empty theory over $\Pi \forall$ ) can be identified with an $L(G)$ structure $\mathbf{M}^{\prime}$ for some ordered abelian group $G$.

Proof. Let $B=\{\|\varphi\|: \varphi \in \tau(M)\}$ and let $S=B \backslash\{0,1\}$. It is readily checked that $B$ is closed under + and $\Rightarrow$, hence $(B,+, \leq, 0,1)$ is itself a BL-chain. Additionally, it follows from $\mathbf{M}$ being a model of $\Pi \forall$ that the ordered abelian semigroup $(S,+, \leq)$ is cancellative and satisfies $a+b<a$ for all $a, b \in S$. Thus, by either Lemma 2.3 of [LS1] or Theorem 4.1 .8 of $[\mathrm{H}], S$ is the set of negative elements of an ordered abelian group $G$. Then $\mathbf{M}$ can be identified with an $L(G)$-structure for any such $G$.

Thus, a formula $\varphi$ is provable from $\Pi \forall$ if and only if $\varphi$ is an $L(G)$ tautology for every ordered abelian group $G$. With our eye on improving this result, we describe a certain class of ordered abelian groups.

Definition 1.6. For $(J,<)$ any linear order, the lexicographic sum $\left(\mathbb{R}^{J},+, \leq\right)$ is the ordered abelian group of functions $f: J \rightarrow \mathbb{R}$ whose support is well-ordered (i.e., $\{j \in J: f(j) \neq 0\}$ is a well-ordered subset of $J$ ). Addition is defined componentwise and the ordering on $\mathbb{R}^{J}$ is lexicographic.

The Hahn embedding theorem states that every ordered abelian group naturally embeds into a lexicographic sum. Moreover, Clifford's proof of this result (see [C]) shows that the embedding can be chosen in a very desirable fashion, which we describe below. 
Definition 1.7. Let $G$ be any ordered abelian group. For $a, b \in G$, we say $a$ and $b$ are equivalent, written $a \sim b$, if $a=b=0$, or $a, b>0$ and there is a positive integer $n$ such that $n a \geq b$ and $n b \geq a$, or $a, b<0$ and there is a positive integer $n$ such that $n a \leq b$ and $n b \leq a$ (the notation $n a$ is shorthand for adding $a$ to itself $n$ times.) For $a, b \in N(G)$ we write $a<<b$ when $a<n b$ for all $n \in \omega$.

It is easily checked that $\sim$ is an equivalence relation on $G$ and that the equivalence classes are convex subsets of $G$. The $\sim$-classes are called the archimedean classes of $G$. Since $G$ is a group, the behavior of $\sim$ on the set of negative elements determines the behavior of $\sim$ on all of $G$. Our notation is slightly nonstandard, as here the elements $a$ and $-a$ are in different archimedean classes whenever $a \neq 0$. Note that $<<$ defines a strict linear order on the archimedean classes of $N(G)$.

It is easily checked that if $f: G \rightarrow H$ is a strict order-preserving homomorphism of ordered abelian groups, then $a \sim b$ if and only if $f(a) \sim f(b)$ for all $a, b \in G$. However, if we want our embedding to preserve suprema and infima, we require an additional property. Specifically, call a strict, order-preserving homomorphism $f: G \rightarrow H$ an archimedean surjection if for every $b \in H$ there is $a \in G$ such that $f(a) \sim b$. Our interest in this notion is given by the following two results.

Lemma 1.8. If $f: G \rightarrow H$ is an archimedean surjection, $X \subset G$, and $a, b \in G$ satisfy $a=\sup (X), b=\inf (X)$, then $f(a)=\sup (f(X))$ and $f(b)=\inf (f(X))$. Moreover, if $X$ is a cofinal (coinitial) subset of $G$, then $f(X)$ is a cofinal (coinitial) subset of $H$.

Proof. By symmetry, inversion, and translation, it suffices to show that if $X$ is a set of positive elements from $G$ and $\inf (X)=0_{G}$, then $\inf (f(X))=0_{H}$. So fix such a set $X \subseteq G$. Since $f$ is strictly orderpreserving every element of $f(X)$ is positive, so $0_{H}$ is a lower bound. Now choose $r \in H, r>0_{H}$. It suffices to find some $a \in X$ such that $f(a)<r$. Before demonstrating this, we establish the following claim:

Claim. For every positive $b \in G$ there is $a \in X$ such that $2 a<b$.

Proof. Fix $b>0$. Choose any $c \in X$ such that $c<b$ and let $d=b-c$. There are now two cases. First, if $2 d \leq b$ then take $a$ to be any element of $X$ less than $d$. Second, if $b<2 d$, then let $e=b-d$ and choose $a \in X$ with $a<e$. Then $2 a<2 e=2 b-2 d \leq b$ and the claim is proved.

Since $f$ is an archimedean surjection we can choose $b \in G$ such that $f(b) \sim r$. Fix a positive integer $n$ so that $f(b) \leq n r$. By iterating the Claim several times we can find an $a \in X$ such that $n a<b$. Hence 
$n f(a)=f(n a)<f(b) \leq n r$, so $f(a)<r$ as required. The 'moreover' clause is proved similarly.

Proposition 1.9. If $f: G \rightarrow H$ is an archimedean surjection and $\mathbf{M}$ is any $L(G)$-structure, then there is a (unique) $L(H)$-structure $\mathbf{M}^{\prime}$ with the same universe as $\mathbf{M}$ that satisfies

$$
f\left(\|\varphi\|_{\mathbf{M}}\right)=\|\varphi\|_{\mathbf{M}^{\prime}}
$$

for all $\tau(M)$-sentences $\varphi$.

Proof. Take $\mathbf{M}^{\prime}$ to have universe $M$ and let $m_{c}^{\mathbf{M}^{\prime}}=m_{c}^{\mathbf{M}}$ for all constants $c \in \tau$. For every predicate symbol $P$, let $r_{P}^{\mathbf{M}^{\prime}}=f\left(r_{P}^{\mathbf{M}}\right)$. One recursively checks that

$$
f\left(\|\varphi\|_{\mathbf{M}}\right)=\|\varphi\|_{\mathbf{M}^{\prime}}
$$

for all $\tau(M)$-sentences $\varphi$, using the previous Lemma to show that quantifiers are well behaved.

The following theorem can be read off from the main result in $[\mathrm{C}]$.

Theorem 1.10 (Clifford's proof of the Hahn embedding theorem). Let $G$ be any ordered abelian group and let $J$ consist of the archimedean classes of the negative elements of $G$ with the induced ordering. Then there is an archimedean surjection $f: G \rightarrow \mathbb{R}^{J}$.

One special case is worth noting. If $G=(0)$ is the trivial group, then $J=\emptyset$ and $\mathbb{R}^{\emptyset}$ is trivial as well. In this case $L\left(\mathbb{R}^{\emptyset}\right)=\{0,1\}$, hence $L\left(\mathbb{R}^{\emptyset}\right)$-structures are classical two-valued structures.

In order to connect the results in this section we introduce the notion of isomorphism of structures in the same predicate language $\tau$. The novelty is that a structure has two distinct sorts (its universe and the associated BL-chain) so an isomorphism itself should be a two-sorted object. Specifically, we say that an $\mathbf{L}$-structure $\mathbf{M}$ is isomorphic to an $\mathbf{L}^{\prime}$-structure $\mathbf{M}^{\prime}$ if there is a BL-chain isomorphism $g: \mathbf{L} \rightarrow \mathbf{L}^{\prime}$ and a bijection $f: M \rightarrow M^{\prime}$ such that

$$
\left\|\varphi\left(f\left(a_{1}\right), \ldots, f\left(a_{n}\right)\right)\right\|_{\mathbf{M}^{\prime}}=g\left(\left\|\varphi\left(a_{1}, \ldots, a_{n}\right)\right\|_{\mathbf{M}}\right)
$$

for all $\tau$-formulas $\varphi\left(x_{1}, \ldots, x_{n}\right)$ and all $\left(a_{1}, \ldots, a_{n}\right) \in M^{n}$. That said, the Corollary below follows immediately from our previous results.

Corollary 1.11. Every model of $\Pi \forall$ is identified with a structure that is isomorphic to an $L\left(\mathbb{R}^{J}\right)$-structure for some (possibly empty) linear $\operatorname{order}(J,<)$.

Corollary 1.12. A formula $\varphi$ is provable from $\Pi \forall$ if and only if $\varphi$ is an $L\left(\mathbb{R}^{J}\right)$-tautology for every linear order $(J,<)$. 


\section{Closed models AND LeXicographic SUMS}

Together with Hájek's Completeness Theorem, Corollary 1.11 tells us that if we want to semantically determine whether a formula is provable from a theory $T$ extending $\Pi \forall$ it suffices to look at structures whose associated BL-chain arise from lexicographic sums. In this section we analyze the sets of tautologies for each of the lexicographic sums $\mathbb{R}^{J}$. In order to compare these sets of tautologies we need a suitable notion of embedding between lexicographic sums. Archimedean surjections are very nice, but unfortunately an archimedean surjection between lexicographic sums $\mathbb{R}^{J}$ and $\mathbb{R}^{K}$ exists only when the linear orders $(J,<)$ and $(K,<)$ are isomorphic. Thus, we both weaken our notion of embedding and strengthen our requirements on the class of 'suitable' structures.

Definition 2.1. A BL-chain embedding is a strict, order-preserving homomorphism $f: \mathbf{L} \rightarrow \mathbf{L}^{\prime}$ of the BL-chains $\mathbf{L}$ and $\mathbf{L}^{\prime}$. Such an embedding respects 0 if, moreover, for every subset $X \subseteq \mathbf{L}$, if $\inf (X)=$ $0_{\mathbf{L}}$ then $\inf (f(X))=0_{\mathbf{L}^{\prime}}$. If $\mathbf{M}$ is an $\mathbf{L}$-structure and $f: \mathbf{L} \rightarrow \mathbf{L}^{\prime}$ is a BL-chain embedding, then $f(\mathbf{M})$ is the $\mathbf{L}^{\prime}$-structure $\mathbf{M}^{\prime}$ with universe $M, m_{c}^{\mathbf{M}^{\prime}}=m_{c}^{\mathbf{M}}$, and $r_{P}^{\mathbf{M}^{\prime}}=f\left(r_{P}^{\mathbf{M}}\right)$.

The reader is cautioned that without extra conditions being placed on either the embedding or the structure, it is possible that the image of a safe L-structure under a BL-chain embedding need not be safe. If $f: G \rightarrow H$ is a strict, order preserving homomorphism of ordered abelian groups, then $f$ extends naturally to a BL-chain embedding (also called $f$ ) from $L(G)$ to $L(H)$ by positing that $f(-\infty)=-\infty$. It is easily checked that this induced embedding respects 0 if and only if the mapping of ordered abelian groups is coinitiality preserving.

Definition 2.2. Fix a BL-chain $\mathbf{L}$ and a predicate language $\tau$.

(1) An $\mathbf{L}$-structure $\mathbf{M}$ is strongly closed $d^{1}$ if for all $\tau(M)$-formulas $\varphi(x)$ with one free variable, there are $a, b \in M$ such that $\|\varphi(a)\|=\|\exists x \varphi(x)\|$ and $\|\varphi(b)\|=\|\forall x \varphi(x)\|$.

(2) An $\mathbf{L}$-structure $\mathbf{M}$ is closed if for all $\tau(M)$-formulas $\varphi(x)$ with one free variable, there are $a, b \in M$ such that $\|\varphi(a)\|=$ $\|\exists x \varphi(x)\|$ and either $\|\varphi(b)\|=\|\forall x \varphi(x)\|$ or $\|\forall x \varphi(x)=0\|$.

Note that if $\mathbf{M}$ is strongly closed then it is closed, and if it is closed then it is safe. The following Lemma is proved by an easy induction on the complexity of $\varphi$ (cf. Proposition 1.9).

\footnotetext{
${ }^{1}$ Strongly closed structures are also called witnessed in the literature, e.g., [H2].
} 
Lemma 2.3. If $\mathbf{M}$ is a closed $\mathbf{L}$-structure and $f: \mathbf{L} \rightarrow \mathbf{L}^{\prime}$ respects 0 , then $\|\varphi\|_{f(\mathbf{M})}=f\left(\|\varphi\|_{\mathbf{M}}\right)$ for all $\tau(M)$-sentences $\varphi$. In particular, $\mathbf{M}$ models $T$ if and only if $f(\mathbf{M})$ models $T$ for any theory $T$.

Similarly, if $\mathbf{M}$ is a strongly closed $\mathbf{L}$-structure, then the conclusions of Lemma 2.3 apply for any BL-chain embedding. This observation yields a slight strengthening of Corollary 1.11. At the end of this section we will achieve a far stronger result.

Lemma 2.4. If $T$ extends $\Pi \forall$ and $T \forall \sigma$, then there is a nonempty ordering $(J,<)$ and an $L\left(\mathbb{R}^{J}\right)$-structure $\mathbf{M}$ that models $T$, yet $\|\sigma\|_{\mathbf{M}}<$ 1. In particular, any such $\mathbb{R}^{J}$ is infinite and divisible.

Proof. In light of Corollary 1.11 we need only consider what happens if there is an $L\left(\mathbb{R}^{\emptyset}\right)$-structure $\mathbf{M}$ that models $T$ with $\|\sigma\|_{\mathbf{M}}<1$ (hence equal to 0 ). Then (trivially) $\mathbf{M}$ is strongly closed. Thus, if $(J,<)$ is arbitrary and $f: L\left(\mathbb{R}^{\emptyset}\right) \rightarrow L\left(\mathbb{R}^{J}\right)$ is any BL-chain embedding (i.e., $f(0)=0$ and $f(1)=1$ ) then $f(\mathbf{M})$ models $T$ and $\|\sigma\|_{f(\mathbf{M})}<1$.

At first blush it seems like the notion of being strongly closed is more natural than that of being closed, but the example below, which exploits the fact that $\rightarrow$ is discontinuous at $(0,0)$, indicates that one cannot prove Theorem 2.6 for such structures.

Example 2.5. Take $\sigma$ to be $(\forall x R \rightarrow S) \rightarrow(\exists x(R \rightarrow S))$ where $R$ and $S$ are unary predicate symbols. Then there are closed structures $\mathbf{M}$ in which $\|\sigma\|_{\mathbf{M}}<1$, yet $\|\sigma\|_{\mathbf{M}}=1$ for every strongly closed structure $\mathbf{M}$.

Theorem 2.6. Let $T$ be any theory extending $\Pi \forall$ and let $\sigma$ be any sentence. If $T \forall \sigma$ then there is a closed model $\mathbf{M}$ of $T$ such that $\|\sigma\|_{\mathbf{M}}<1$. Furthermore, if the language is countable then $\mathbf{M}$ can be chosen to be countable as well.

Before proving Theorem 2.6 we state and prove two proof-theoretic lemmas about the axiom system $\Pi \forall$. In keeping with the spirit of the paper, our proofs of these facts will be model theoretic in nature. However, in $[\mathrm{M}]$ the second author proves these lemmas directly from the axiom system.

Lemma 2.7. Suppose that $T$ is a theory extending $\Pi \forall, \sigma$ is a sentence, $c$ is a constant symbol that does not appear in either $T$ or $\sigma$, and $T \cup$ $\{\exists x \varphi(x) \rightarrow \varphi(c)\} \vdash \sigma$. Then $T \vdash \sigma$.

Proof. Let $\tau$ denote the language of $T \cup\{\sigma\}$ and let $\tau_{c}=\tau \cup\{c\}$. Let $\mathbf{M}$ be an $L\left(\mathbb{R}^{J}\right)$-structure in the language of $\tau$ that models $T$ in which $J \neq \emptyset$. In particular, $\mathbb{R}^{J}$ is infinite and divisible as an abelian group. 
Because of Lemma 2.4, in order to show that $T \vdash \sigma$ it suffices to show that $\|\sigma\|_{\mathbf{M}}=1$. Since $L\left(\mathbb{R}^{J}\right)$ is infinite and divisible, 1 is an accumulation point of $\mathbb{R}^{J}$, so it suffices to prove that $\|\sigma\|_{\mathbf{M}} \geq \epsilon$ for every $\left.\epsilon \in L\left(\mathbb{R}^{J}\right)\right), \epsilon<1$.

For each $a \in M$, let $\mathbf{M}_{a}$ denote the expansion of $\mathbf{M}$ to a structure in the language $\tau_{c}$ formed by setting $m_{c}^{\mathbf{M}_{a}}=a$. As notation, let $\theta(y)$ abbreviate $\exists x \varphi(x) \rightarrow \varphi(y)$. Since $T \cup \theta(c) \vdash \sigma$, it follows from the Deduction theorem that we can fix a positive integer $k$ such that $T \vdash$ $\theta(c)^{k} \rightarrow \sigma$. Since $\mathbf{M}$ is a model of $T$, each $\mathbf{M}_{a}$ is a model of $T$, hence $\left\|\theta(c)^{k} \rightarrow \sigma\right\|_{\mathbf{M}_{a}}=1$ for all $a \in M$. Reflecting back to $\mathbf{M}$, this implies $\left\|\theta(a)^{k} \rightarrow \sigma\right\|_{\mathbf{M}}$ for all $a \in M$, hence

$$
k\|\theta(a)\|_{\mathbf{M}} \leq\|\sigma\|_{\mathbf{M}} \quad \text { for all } a \in M
$$

Now fix an $\epsilon<1$. From our comments above, it suffices to show that $k\|\theta(a)\|_{\mathbf{M}} \geq \epsilon$ for some $a \in M$.

But $\|\exists x \varphi(x)\|_{\mathbf{M}}=\sup \left\{\|\varphi(a)\|_{\mathbf{M}}: a \in M\right\}$, so there is some $a \in M$ so that $\|\varphi(a)\|_{\mathbf{M}} \geq\|\exists x \varphi(x)\|_{\mathbf{M}}+\epsilon / k$ (this makes sense since $\mathbb{R}^{J}$ is divisible). It follows that $k\|\theta(a)\|_{\mathbf{M}} \geq \epsilon$ and the lemma is proved.

Lemma 2.8. Suppose that $T$ is a theory extending $\Pi \forall, \sigma$ is a sentence, $c$ is a constant symbol that does not appear in either $T$ or $\sigma$, and both $T \cup\{\forall x \varphi(x) \rightarrow 0\} \vdash \sigma$ and $T \cup\{\varphi(c) \rightarrow \forall x \varphi(x)\} \vdash \sigma$. Then $T \vdash \sigma$.

Proof. Fix $\tau, \tau_{c}$ and $\mathbf{M}$ as in the proof of the Lemma 2.7. As before, it suffices to prove that $\|\sigma\|_{\mathbf{M}}=1$. First, if $\|\forall x \varphi(x)\|_{\mathbf{M}}=0$, then $\mathbf{M}$ would be a model of $T \cup\{\forall x \varphi(x) \rightarrow 0\} \vdash \sigma$ and $\|\sigma\|_{\mathbf{M}}=1$ by our hypothesis. So we assume that $\|\forall x \varphi(x)\|_{\mathbf{M}} \neq 0$. As notation, let $\psi(y)$ abbreviate $\varphi(y) \rightarrow \forall x \varphi(x)$ and, for each $a \in M$, let $\mathbf{M}_{a}$ denote the expansion of $\mathbf{M}$ satisfying $m_{c}^{\mathbf{M}_{a}}=a$. As in the proof of Lemma 2.7 choose $k$ so that $T \vdash \psi(c)^{k} \rightarrow \sigma$. As in that argument, by considering each of the $\mathbf{M}_{a}$ 's and reflecting back to $\mathbf{M}$, we obtain that

$$
k\|\psi(a)\|_{\mathbf{M}} \leq\|\sigma\|_{\mathbf{M}} \quad \text { for all } a \in M
$$

Now fix an $\epsilon<1$.

Since $\|\forall x \varphi(x)\|_{\mathbf{M}}=\inf \{\|\varphi(a)\|: a \in M\}$ and $\|\forall x \varphi(x)\|_{\mathbf{M}} \neq 0$, there is some $a \in M$ so that $\|\forall x \varphi(x)\|_{\mathbf{M}} \geq\|\varphi(a)\|+\epsilon / k$ (again $\mathbb{R}^{J}$ is divisible). As before, this implies that $k\|\psi(a)\|_{\mathbf{M}} \geq \epsilon$. Since $\|\sigma\|_{\mathbf{M}} \geq$ $\|\psi(a)\|_{\mathbf{M}}$ and $\epsilon<1$ was arbitrary, it follows that $\|\sigma\|_{\mathbf{M}}=1$.

Proof of Theorem 2.6. We follow the proof of Hájek's Completeness Theorem (Theorem 5.2.9 of $[\mathrm{H}]$ ). Therein, given $T$ and $\sigma$ in the language $\tau$, he first augments $\tau$ by adding one new constant symbol for each formula in the original language. Then, in Lemma 5.2.7 he iteratively constructs an extension $T^{\prime}$ of $T$ in this expanded language that 
is complete (i.e., for every pair $(\varphi, \psi)$ of sentences, either $T \vdash(\varphi \rightarrow \psi)$ or $T \vdash(\psi \rightarrow \varphi)$ ) and Henkin (i.e., for every formula $\varphi(x)$ with one free variable if $T \forall \forall x \varphi(x)$ then there is a constant symbol $c$ such that $T \forall \varphi(c))$ while still preserving that $T^{\prime} \forall \sigma$. In our context, we do precisely the same thing, but by iteratively using Lemmas 2.7 and 2.8 to handle each formula $\varphi(x)$ we additionally require that $T^{\prime}$ satisfy two additional requirements:

- For all $\varphi(x)$ in the expanded language there is a constant symbol $c$ such that $T^{\prime} \vdash \exists x \varphi(x) \rightarrow \varphi(c)$ and

- For all $\varphi(x)$ in the expanded language either $T^{\prime} \vdash \forall x \varphi(x) \rightarrow \overline{0}$ or there is a constant symbol $c$ such that $T^{\prime} \vdash \varphi(c) \rightarrow \forall x \varphi(x)$.

Then, just as in Hájek, one can canonically construct a structure $\mathbf{M}$ whose universe consists of the constant symbols of the expanded language. In his context $\mathbf{M}$ was safe, $\mathbf{M}$ model of $T^{\prime}$ (hence the reduct to the original language is a model of $T$ ) and $\|\sigma\|_{\mathbf{M}}<1$. It is easy to see that with the addition of the two extra properties noted above, $\mathbf{M}$ is closed.

We close this section by indicating a general construction and then an application of it which asserts the existence of a 'universal' BL-chain in the context of predicate product logic.

Fix a language $\tau$. Given any BL-chain $\mathbf{L}$ and any $\mathbf{L}$-structure $\mathbf{M}=$ $\left(M, m_{c}, r_{P}\right)$, we form a first-order, two-valued structure that encodes all of this data. Specifically, let

$$
\mathfrak{M}=\left(M, L,+, \leq, 0,1, m_{c}, r_{P}\right)
$$

be the two-sorted structure in which $\{+, \leq, 0,1\}$ refer to the BL-chain, each $m_{c}$ points to an element in the $M$-sort, and $r_{P}: M^{n} \rightarrow L$ is the map described by $\mathbf{M}$ for each $n$-ary $P \in \tau$.

Many of the notions discussed in this paper are first-order in this language. If $\mathfrak{M}^{\prime}=\left(M^{\prime}, L^{\prime}, \ldots\right)$ is elementarily equivalent to the structure $\mathfrak{M}$ defined above, then $\mathbf{L}^{\prime}=\left(L^{\prime},+, \leq, 0,1\right)$ is a BL-chain and $\mathfrak{M}^{\prime}$ describes an $\mathbf{L}^{\prime}$-structure $\mathbf{M}^{\prime}$ with universe $M^{\prime}$. One can check that $\mathbf{M}$ is safe (resp. closed) if and only if $\mathbf{M}^{\prime}$ is safe (closed). Using the algebraic characterization of such semigroups in Proposition 1.5, the BL-chain $\mathbf{L}$ is equal to $L(G)$ for some ordered abelian group if and only if $\mathbf{L}^{\prime}=L\left(G^{\prime}\right)$ for some group $G^{\prime}$. Furthermore, if $\mathfrak{M}$ is an elementary substructure of $\mathfrak{M}^{\prime}$ (in the usual first-order sense) one can inductively argue that $\|\varphi\|_{\mathbf{M}}=\|\varphi\|_{\mathbf{M}^{\prime}}$ for all $\tau(M)$-sentences $\varphi$.

Theorem 2.9. If $\tau$ is countable, $T \supseteq \Pi \forall$ and $T \forall \sigma$, then there is a countable, closed $L\left(\mathbb{R}^{\mathbb{Q}}\right)$-structure $\mathbf{M}$ that models $T$ but does not model 
$\sigma$. In particular, a sentence $\sigma$ is an $L\left(\mathbb{R}^{\mathbb{Q}}\right)$-tautology if and only if $\sigma$ is provable from $\Pi \forall$.

Proof. Fix $\tau, T$, and $\sigma$ as in the hypotheses. By Lemma 2.4 there is a nonempty $(J,<)$ and a closed $L\left(\mathbb{R}^{J}\right)$-structure $\mathbf{M}$ that models $T$, but $\|\sigma\|_{\mathbf{M}}<1$. Form the first-order structure $\mathfrak{M}=\left(M, L\left(\mathbb{R}^{J}\right), \ldots\right)$ described above. Let $\mathfrak{M}_{0}$ be any countable elementary substructure of $\mathfrak{M}$. Note that by elementarity the BL-chain associated to $\mathfrak{M}_{0}$ is equal to $L(G)$ for some infinite ordered abelian group $G$.

We now form an increasing elementary chain of countable (firstorder) structures $\mathfrak{M}_{0} \preceq \mathfrak{M}_{1} \preceq \ldots$ as follows: Given $\mathfrak{M}_{k}$ consider the type $p(x)$ stating that $x$ is in the $L$-sort, $x>0$, but for any element $c$ in the $L$-sort of $\mathfrak{M}_{k}, x<n c$ for all positive integers $n . p$ is clearly consistent since the $L$-sort of $\mathfrak{M}_{k}$ has the form $L\left(G_{k}\right)$ for some infinite ordered abelian group $G_{k}$. So choose $\mathfrak{M}_{k+1}$ to be any countable elementary extension of $\mathfrak{M}_{k}$ that realizes $p$.

Let $\mathfrak{M}^{*}=\left(M^{*}, L^{*}, \ldots\right)$ be the union of the chain, let $\mathbf{L}^{*}=\left(L^{*},+, \leq\right.$ $, 0,1)$, and let $\mathbf{M}^{*}$ be the $\mathbf{L}^{*}$-structure coded by $\mathfrak{M}^{*}$. Since $\mathfrak{M}_{0} \preceq \mathfrak{M}^{*}$, $\mathbf{M}^{*}$ is a closed model of $T$ with $\|\sigma\|_{\mathbf{M}^{*}}<1$. Also, $\mathbf{L}^{*}=L\left(G^{*}\right)$ for some ordered abelian group $G^{*}$. As well, our construction guarantees that there is no smallest archimedean class of the negative elements of $G^{*}$. By Theorem 1.10 we can choose $(J,<)$ and an archimedean surjection $f: G^{*} \rightarrow \mathbb{R}^{J}$. It follows from Proposition 1.9 that $f\left(\mathbf{M}^{*}\right)$ is closed, is a model of $T$, and $\|\sigma\|_{f\left(\mathbf{M}^{*}\right)}<1$. Since $G^{*}$ is countable, $J$ is countable. Furthermore, $J$ has no smallest element. Thus, there is an order-preserving coinitial map $g: J \rightarrow \mathbb{Q}$. This map induces a BL-chain embedding $g^{\prime}: L\left(\mathbb{R}^{J}\right) \rightarrow L\left(\mathbb{R}^{\mathbb{Q}}\right)$. Since $f\left(\mathbf{M}^{*}\right)$ is a closed $L\left(\mathbb{R}^{J}\right)$-structure, it follows from our construction and Lemma 2.3 that $g^{\prime}\left(f\left(\mathbf{M}^{*}\right)\right)$ is a closed $L\left(\mathbb{R}^{\mathbb{Q}}\right)$-structure that models $T$ with $\|\sigma\|<1$.

\section{INITIALLY DENSE LINEAR ORDERINGS}

In this section we obtain a dichotomy among the sets of $L\left(\mathbb{R}^{J}\right)$ tautologies that is related to the order type of $(J,<)$.

Definition 3.1. A linear ordering $(J,<)$ is initially dense if there is a coinitiality preserving embedding $f:(\mathbb{Q},<) \rightarrow(J,<)$.

It is readily checked that if $(J,<)$ is countable, then $J$ is not initially dense if and only if there is some $a \in J$ such that $\{b \in J: b \leq a\}$ is scattered. The following theorem indicate that the BL-chain $L\left(\mathbb{R}^{J}\right)$ is universal in a strong sense whenever $J$ is initially dense. 
Theorem 3.2. Suppose that $(J,<)$ is initially dense. If $\sigma$ is any sentence and $T \supseteq \Pi \forall$ is any theory that has a model that does not model $\sigma$, then there is a closed $L\left(\mathbb{R}^{J}\right)$-model of $T$ that does not model $\sigma$. In particular, a sentence $\sigma$ is an $L\left(\mathbb{R}^{J}\right)$-tautology if and only if $\sigma$ is provable from $\Pi \forall$.

Proof. Fix $T$ and $\sigma$ as in the hypothesis. By Theorem 2.9 there is a closed $L\left(\mathbb{R}^{\mathbb{Q}}\right)$-structure $\mathbf{M}$ that models $T$ but does not model $\sigma$. Fix a coinitiality preserving embedding of $(\mathbb{Q},<)$ into $(J,<)$. This embedding naturally induces a coinitiality preserving ordered group homomorphism $f: \mathbb{R}^{\mathbb{Q}} \rightarrow \mathbb{R}^{J}$ of lexicographic sums, which in turn yields a BL-chain embedding $f: L\left(\mathbb{R}^{\mathbb{Q}}\right) \rightarrow L\left(\mathbb{R}^{J}\right)$ that respects 0 . Since $\mathbf{M}$ is closed, Lemma 2.3 implies that $f(\mathbf{M})$ is the desired structure.

By contrast, Montagna [Mo] proves that the set of tautologies of $L\left(\mathbb{R}^{1}\right)$ is not arithmetical. Here we extend his method to show that the set of $L\left(\mathbb{R}^{J}\right)$-tautologies are not arithmetical whenever $J$ is countable but not initially dense. We begin our analysis with a construction that appears in $[\mathrm{Mo}]$.

Let $\tau$ be a finite, relational vocabulary (for simplicity we do not allow $\tau$ to have any constant symbols in this discussion) containing a distinguished binary relation $E$. For any $\tau$-formula $\varphi$, let $\varphi^{\circ}$ denote the $\tau$-formula in which every relation symbol $R \in \tau$ is replaced by $\neg \neg R$. Note that if $\mathbf{M}$ is any model of $\Pi \forall$ then $\left\|R^{\circ}\left(a_{1}, \ldots, a_{n}\right)\right\|^{\mathbf{M}} \in\{0,1\}$ for any $a_{1}, \ldots a_{n}$ from $M$ and $\left\|R^{\circ}\left(a_{1}, \ldots, a_{n}\right)\right\|_{\mathbf{M}}=1$ if and only if $\left\|R\left(a_{1}, \ldots, a_{n}\right)\right\|_{\mathbf{M}}>0$. It follows by induction on the complexity of $\varphi$ that $\|\varphi(\bar{a})\|_{\mathbf{M}} \in\{0,1\}$ for any $\tau$-formula $\varphi$ and any tuple $\bar{a}$ from $M$. Moreover, the interpretation of quantifiers is as in 2-valued logic, e.g., $\left\|\exists x \varphi^{\circ}(x, \bar{a})\right\|_{\mathbf{M}}=1$ if and only if $\left\|\varphi^{\circ}(b, \bar{a})\right\|_{\mathbf{M}}=1$ for some $b \in M$.

Let $\operatorname{Quot}(\tau)$ denote the conjunction of (finitely many) axioms asserting that $E^{\circ}$ is an equivalence relation and that

$\forall x_{1}, \ldots, x_{n} \forall y_{1} \ldots, y_{n}\left(\bigwedge_{i} E^{\circ}\left(x_{i}, y_{i}\right) \wedge R^{\circ}\left(x_{1}, \ldots, x_{n}\right) \rightarrow R^{\circ}\left(y_{1}, \ldots, y_{n}\right)\right)$

for each relation symbol $R \in \tau$. Note that $\|\sigma\|_{\mathbf{M}} \in\{0,1\}$ for each $\sigma \in Q \operatorname{uot}(\tau)$ and any model $\mathbf{M}$ of $\Pi \forall$. We call a model $\mathbf{M}$ of $\Pi \forall \tau$ quotientable (with respect to the distinguished relation $E$ ) if $\|\sigma\|_{\mathbf{M}}=1$ for all $\sigma \in Q u o t(\tau)$. As the name suggests, it is easily checked that if $\mathbf{M}$ is $\tau$-quotientable, then $E^{\circ}$ is an equivalence relation on $M$. As notation, for each $a \in M$, let $[a]=\left\{b \in M:\left\|E^{\circ}(a, b)\right\|_{\mathbf{M}}=1\right\}$ and let $M^{\circ}=\{[a]: a \in M\}$. Furthermore, we can define an $L(\{0,1\})$-structure $\mathbf{M}^{\circ}$ with universe $M^{\circ}$ by positing that $R^{\mathbf{M}^{\circ}}\left(\left[a_{1}\right], \ldots,\left[a_{n}\right]\right)$ holds if and only if $\left\|R^{\circ}\left(a_{1}, \ldots, a_{n}\right)\right\|_{\mathbf{M}}=1$. (The axioms of $Q u o t(\tau)$ guarantee that 
this is well-defined.) It follows by induction on the complexity of $\varphi$ that

$$
\mathbf{M}^{\circ} \models \varphi\left(\left[a_{1}\right], \ldots,\left[a_{n}\right]\right) \quad \text { if and only if }\left\|\varphi^{\circ}\left(a_{1} \ldots, a_{n}\right)\right\|_{\mathbf{M}}=1
$$

for any choice of representatives for $\left[a_{1}\right], \ldots,\left[a_{n}\right]$.

Let $\tau_{a}=\{E, L, S, Z, A, P\}$ denote the (relational) language of arithmetic. The relations $E, L, S$ are binary and their intended interpretations are equality, strict less than, and the graph of the successor function. $Z$ is unary and is intended to denote the class of zero elements, while $A$ and $P$ are ternary and are intended to represent the graphs of addition and multiplication. Let $\mathfrak{N}$ denote the standard $\{0,1\}$-model of arithmetic in the vocabulary $\tau_{a}$, i.e., the universe of $\mathfrak{N}$ is $\omega$ and each of the relations are given their intended interpretations.

Next, let $Q^{*}$ be the finite set of $\tau_{a}$-sentences $\operatorname{Quot}\left(\tau_{a}\right)$, together with modified versions of each axiom of Robinson's $Q$. The modifications are two-fold. First, they need to be written in our relational language $\tau_{a}$. Second, we replace each relation symbol $R$ by $R^{\circ}$. So, for example, the axiom $\forall x(x<S(x))$ becomes $\forall x \forall y\left(S^{\circ}(x, y) \rightarrow L^{\circ}(x, y)\right)$.

It is easily checked that if $\mathbf{M}$ is any model of $Q^{*}$ then the two-valued structure $\mathbf{M}^{\circ}$ (which is well-defined since $\mathbf{M}$ is quotientable) is a model of Robinson's $Q$. Note that Robinson's $Q$ is strong enough to determine the 'standard part' of any two-valued model of $Q$. In particular, if $\mathbf{M}^{\circ} \models Q$ is 'standard', i.e., every class is an iterated successor of the zero class, then $\mathbf{M}^{\circ}$ is $\tau_{a}$-isomorphic to $\mathfrak{N}$.

Let $\tau_{U}=\tau_{a} \cup\{U\}$, where $U$ is a unary predicate. We will be interested in structures $\mathbf{M}$ in the vocabulary $\tau_{U}$ and their reducts to $\tau_{a}$.

Definition 3.3. A structure $\mathbf{M}$ modelling $\Pi \forall$ in the vocabulary $\tau_{U}$ has a standard arithmetical part if $\|\sigma\|_{\mathbf{M}}=1$ for all $\sigma \in Q^{*}$ and the associated two-valued structure $\mathbf{M}^{\circ}$ is $\tau_{a}$-isomorphic to $\mathfrak{N}$.

Let $\Psi$ denote $\tau_{U}$-sentence that is the conjunction of $Q^{*}$ with the following five sentences:

- $\psi_{1}:=\forall x \neg \neg U(x)$;

- $\psi_{2}:=\neg \forall x U(x)$;

- $\psi_{3}:=\forall x \forall y\left(E^{\circ}(x, y) \wedge U(x) \rightarrow U(y)\right)$;

- $\psi_{4}:=\forall x \forall y\left(L^{\circ}(x, y) \wedge U(y) \rightarrow U(x)\right)$;

- $\psi_{5}:=\forall x \forall y\left(S^{\circ}(x, y) \wedge U(y) \rightarrow(U(x) \& U(x) \& U(x))\right)$.

Lemma 3.4. If $(J,<)$ is countable then there is an $L\left(\mathbb{R}^{J}\right)$-model of $\Psi$.

Proof. Fix any countable $J$. Since $\mathbb{R}^{J}$ has countable coinitiality, we can choose elements $\left\langle b_{n}: n \in \omega\right\rangle$ from $\mathbb{R}^{J}$ such that $\left\{b_{n}: n \in \omega\right\}$ 
are coinitial and such that $b_{n+1}<3 b_{n}$ for each $n \in \omega$. Let $\mathbf{M}$ be the structure in the vocabulary $\tau_{U}$ with universe $\omega$ in which each of the relations in $L_{a}$ is given its 'standard' interpretation and $\|U(n)\|_{\mathbf{M}}=b_{n}$ for each $n \in \omega$.

Proposition 3.5. Suppose that $(J,<)$ is countable but not initially dense and $\mathbf{M}$ is any $L_{U}\left(\mathbb{R}^{J}\right)$-model of $\Pi \forall$ such that $\|\Psi\|_{\mathbf{M}}>0$. Then $\mathbf{M}$ has a standard arithmetical part.

Proof. Fix such a $(J,<)$ and $\mathbf{M}$. To ease notation, we write $\|\cdot\|$ in place of $\|\cdot\|_{\mathbf{M}}$ throughout the proof of this lemma. Let $\|\Psi\|=\gamma$. Since $\gamma \neq 0, \gamma \in N\left(\mathbb{R}^{J}\right)$. Since $\|\sigma\| \in\{0,1\}$ for each $\sigma \in Q^{*}$, it follows that $\|\sigma\|=1$ for each $\sigma \in Q^{*}$. Thus, the reduct of $\mathbf{M}$ is $\tau_{a}$-quotientable. As well, since $\left\|\psi_{1}\right\|>0\|U(a)\| \neq 0$, hence $\|U(a)\| \in N\left(\mathbb{R}^{J}\right)$ for all $a \in M$. Since $\left\|\psi_{2}\right\| \neq 0,\{\|U(a)\|: a \in M\}$ is coinitial in $N\left(\mathbb{R}^{J}\right)$. Since $\left\|\psi_{i}\right\| \geq \gamma$ for $i \in\{3,4,5\},\|U(b)\| \leq\|U(a)\|-\gamma$ whenever either $E^{\circ}(a, b)$ or $L^{\circ}(a, b)$ hold, and

$$
\|U(b)\| \leq 3\|U(a)\|-\gamma
$$

whenever $S^{\circ}(a, b)$ holds. (Recall that $\gamma$ is a negative element of $\mathbb{R}^{J}$.) Fix an element $c \in M$ such that $\|U(c)\| \leq 2 \gamma$. It follows from (3) that

$$
\|U(b)\| \leq 2\|U(a)\|
$$

whenever $L^{\circ}(c, a)$ and $S^{\circ}(a, b)$ hold.

Now assume by way of contradiction that $\mathbf{M}^{\circ} \neq \mathfrak{N}$. Thus $\mathbf{M}^{\circ} \models Q$, but has nonstandard elements. By iterating (4),

$$
\|U(b)\|<<\|U(a)\|
$$

whenever $L^{\circ}(c, a)$ and $L^{\circ}(a, b)$ hold, and $[b]-[a]$ is nonstandard. That is, $\|U(b)\|$ is in a strictly smaller archimedean class than $\|U(a)\|$. This fact, together with the fact that $\{\|U(a)\|: a \in M\}$ is coinitial in $\mathbb{R}^{J}$ imply that $\mathbb{R}^{J}$ has no smallest archimedean class, i.e., $J$ has no least element.

Fix $c^{*} \in M$ such that $L^{\circ}\left(c, c^{*}\right)$ and $\left\|U\left(c^{*}\right)\right\|<<\gamma$. Since $\beta+\gamma \sim \beta$ whenever $\beta<<\gamma,\left\|\psi_{3}\right\| \geq \gamma$ implies $\|U(a)\| \sim\left\|U\left(a^{\prime}\right)\right\|$ whenever $L^{\circ}\left(c^{*}, a\right), L^{\circ}\left(c^{*}, a^{\prime}\right)$ and $E^{\circ}\left(a, a^{\prime}\right)$. That is, the archimedean class of $\|U(a)\|$ depends only on $[a]$. As well, suppose that $L^{\circ}\left(c^{*}, a\right), L^{\circ}(a, b)$, and $[b]-[a]$ is a nonstandard element of $\mathbf{M}^{\circ}$. Let $d$ be any element in the $E^{\circ}$-class of $([a]+[b]) / 2\left(\right.$ which exists since $\left.\mathbf{M}^{\circ} \models Q\right)$. Then $[b]-[d]$ and $[d]-[a]$ are nonstandard elements and (5) yields

$$
\|U(b)\|<<\|U(d)\|<<\|U(a)\|
$$


We will obtain a contradiction by constructing a coinitiality preserving embedding $f: \mathbb{Q} \rightarrow J$. Let $D=\left\{n / 2^{m}: n \in \omega \backslash\{0\}, m \in \omega\right\}$ denote the positive dyadics. We will construct an embedding $g: D \rightarrow M$ such that $L^{\circ}\left(c^{*}, f(d)\right)$ and $\left\|U\left(d^{\prime}\right)\right\|<<\|U(d)\|$ for all $d<d^{\prime}$ from $D$ and $\{\|U(d)\|: d \in D\}$ is coinitial in $\mathbb{R}^{J}$. Once we have such a $g$, then $f$ can be obtained by composing an isomorphism between $(\mathbb{Q},<)$ and $(D,<)$ with $g$. Since $\mathbf{M}^{\circ}$ is nonstandard and $(J,<)$ is countable with no minimal element we can find $\left\{a_{n}: n \in \omega\right\}$ from $M$ such that $L^{\circ}\left(c^{*}, a_{0}\right), L^{\circ}\left(a_{n}, a_{n+1}\right),\left\|U\left(a_{n+1}\right)\right\|<<\left\|U\left(a_{n}\right)\right\|$ for all $n$ and $\left\{\left\|U\left(a_{n}\right)\right\|: n \in \omega\right\}$ is coinitial in $\mathbb{R}^{J}$. We begin our construction of $g$ by letting $g(n)=a_{n}$. Now suppose $\left\{g\left(n / 2_{l}\right): n \in \omega, l \leq m\right\}$ have been defined. Fix an odd $n \in \omega$, say $n=2 k-1$. Let $d_{k}$ be any element of the $E^{\circ}$-class of $\left(\left[g\left(k / 2^{m}\right)\right]+\left[g\left((k+1) / 2^{m}\right)\right]\right) / 2$. It follows from (6) that $\left\|U\left(g\left((k+1) / 2^{m}\right)\right)\right\|<<\left\|U\left(d_{k}\right)\right\|<<\left\|g\left(k / 2^{m}\right)\right\|$, so let $g\left(n / 2^{m+1}\right)=d_{k}$.

Definition 3.6. For $\sigma$ any sentence in $\tau_{a}$, let $\sigma^{*}$ denote the $\tau_{U}$-sentence $\Psi \rightarrow \sigma^{\circ}$.

Theorem 3.7. If $(J,<)$ is countable but not initially dense, then the set of $L\left(\mathbb{R}^{J}\right)$-tautologies in the vocabulary $\tau_{U}$ is not arithmetical.

Proof. Fix any countable $(J,<)$ that is not initially dense. We argue that for any $\tau_{a}$-sentence $\sigma, \sigma^{*}$ is an $L\left(\mathbb{R}^{J}\right)$-tautology if and only if $\mathfrak{N} \models \sigma$ (in the usual two-valued sense). The Theorem follows immediately from this by Tarski's Theorem and the recursiveness of the $\operatorname{map} \sigma \mapsto \sigma^{*}$.

First, suppose that $\sigma^{*}$ is an $L\left(\mathbb{R}^{J}\right)$-tautology. By Lemma 3.4 we can choose $\mathbf{M}$ such that $\|\Psi\|_{\mathbf{M}}>0$. By Proposition $3.5 \mathbf{M}$ is $\tau_{a^{-}}$ quotientable and $\mathbf{M}^{\circ} \cong \mathfrak{N}$. Since $\sigma^{*}$ is an $L\left(\mathbb{R}^{J}\right)$-tautology and $\|\Psi\|_{\mathbf{M}}>$ 0 , $\left\|\sigma^{\circ}\right\|_{\mathbf{M}}>0$. But, as noted earlier, this implies $\left\|\sigma^{\circ}\right\|_{\mathbf{M}}=1$, hence $\mathbf{M}^{\circ} \models \sigma^{\circ}$. Since $\neg \neg \varphi$ is equivalent to $\varphi$ in the class of two-valued structures and $\mathbf{M}^{\circ} \cong \mathfrak{N}, \mathfrak{N} \models \sigma$.

Conversely, suppose $\mathfrak{N} \models \sigma$. Let $\mathbf{M}$ be any $L\left(\mathbb{R}^{J}\right)$-structure with vocabulary $\tau_{U}$. We argue that $\left\|\sigma^{*}\right\|_{\mathbf{M}}=1$. This is immediate if $\|\Psi\|_{\mathbf{M}}=0$, so assume $\|\Psi\|_{\mathbf{M}}>0$. Then, again by Proposition 3.5, $\mathbf{M}$ is $\tau_{a}$-quotientable and $\mathbf{M}^{\circ} \cong \mathfrak{N}$. Thus, $\mathbf{M}^{\circ} \models \sigma^{\circ}$, so $\left\|\sigma^{\circ}\right\|_{\mathbf{M}}=1$, which implies $\left\|\sigma^{*}\right\|_{\mathbf{M}}=1$.

Remark 3.8. Note that the proofs of both Proposition 3.5 and Theorem 3.7 only require that $(J,<)$ have countable coinitiality (and not initially dense). 
Corollary 3.9. The following are equivalent for a countable linear or$\operatorname{der}(J,<)$.

(1) For all countable vocabularies $\tau$, a sentence $\sigma$ is an $L\left(\mathbb{R}^{J}\right)$ tautology if and only if $\sigma$ is provable from $\Pi \forall$;

(2) For all finite vocabularies $\tau$, the set of $L\left(\mathbb{R}^{J}\right)$-tautologies is arithmetic;

(3) $(J,<)$ is initially dense.

Proof. Immediate by Theorems 3.2 and 3.7 .

Corollary 3.10. Let $\sigma$ be any $\tau_{a}$-sentence such that $\mathfrak{N} \models \sigma$, but $Q \forall \sigma$ (in the usual proof theory of first-order logic). Let $(J,<)$ be a countable linear order. Then $\sigma^{*}$ is an $L\left(\mathbb{R}^{J}\right)$-tautology if and only if $(J,<)$ is not initially dense.

\section{REFERENCES}

[C] A. H. Clifford, A note on Hahn's theorem on ordered abelian groups, Proc. $A M S$ 5(1954) 860-863.

[H] P. Hájek, Metamathematics of Fuzzy Logic, Kluwer Academic Publishers, Boston, 1998.

[H2] P. Hájek, Making fuzzy description logic more general, Fuzzy Sets and Systems, 154(2005), no. 1, 1-15.

[LS1] M.C. Laskowski, and Y.V. Shashoua, A classification of BL-algebras, Fuzzy Sets and Systems, 131(2002), no. 3, 271-282.

[LS2] M.C. Laskowski, and Y.V. Shashoua, Generalized ordinal sums and the decidability of BL-chains, Algebra Universalis, 52(2004), 137-153.

[M] S. Malekpour, PhD thesis, University of Maryland, 2004.

[Mo] F. Montagna, Three complexity problems in quantified fuzzy logic, Studia Logica 68(2001) 143-152.

Department of Mathematics, University of Maryland, College Park, MD 20742

E-mail address: mcl@math.umd.edu

Department of Mathematics, University of Wisconsin, Madison, MadiSON, WI 53706

E-mail address: malekpou@math.wisc.edu 